\title{
CDISC Therapeutic Area User Guide Name and Version
}

National Cancer Institute

\section{Source}

National Cancer Institute. CDISC Therapeutic Area User Guide Name and Version. NCI

Thesaurus. Code C156602.

The name and version of the CDISC therapeutic area user guide that is being used in the study submission. 